\title{
PERTURBATIVE QUANTUM CHROMODYNAMICS
}




\section{Published}

Vol. 1 - High Energy Electron-Positron Physics (eds. A. Ali and P. Soding)

Vol. 2 - Hadronic Multiparticle Production (ed. P. Carruthers)

Vol. 3 - CP Violation (ed. C. Jarlskog)

Vol. 4- Proton-Antiproton Collider Physics (eds. G. Altarelli and L. Di Lella)

Vol. 5- Perturbative OCD (ed. A. H. Mue/ler)

\section{Forthcoming}

Vol. 6- Quark Gluon Plasma (ed. R. C. Hwa)

Vol. 7 - Quantum Electrodynamics (ed. T. Kinoshita)

Vol. 8 - Interactions Between Elementary Particle Physics and Cosmology (ed. E. Kolb) 
Advanced Series on

Directions in High Energy Physics — Vol. 5

\title{
PERTURBATIVE QUANTUM CHROMODYNAMICS
}

Editor:

\author{
A. H. Mueller
}


Published by

World Scientific Publishing Co. Pte. Ltd., P O Box 128, Farrer Road, Singapore 9128

USA office: 687 Hartwell Street, Teaneck, NJ 07666

UK office: 73 Lynton Mead, Totteridge, London N20 8DH

Library of Congress Cataloging-in-Publication data is available.

\section{PERTURBATIVE QUANTUM CHROMODYNAMICS}

Copyright $\odot 1989$ by World Scientific Publishing Co Pte Ltd.

All rights reserved. This book, or parts thereof, may not be reproduced in any forms or by any means, electronic or mechanical, including photocopying, recording or any information storage and retrieval system now known or to be invented, without written permission from the Publisher.

ISBN 9971-50-564-9

$9971-50-565-7$ (pbk)

ISSN 0218-0324

Printed in Singapore by Utopia Press. 


\section{FOREWORD}

With the discovery of asymptotic freedom in 1973 Quantum Chromodynamics (QCD) was born. It was soon realized that the study of nonperturbative effects would be crucial in order to understand color confinement, chiral symmetry breaking and, of course, to achieve a quantitative understanding of bound states and low energy dynamics. It was also realized, right from the start, that the rather rich structure of QCD perturbation theory could be seen in hadronic processes involving a high momentum transfer, that is, in hard processes. QCD is widely viewed to be the correct theory of the strong interactions, mainly because of the success which has been achieved in predicting and describing such hard processes.

The road has not been easy. It has been necessary to develop an extensive theoretical apparatus in order to relate properties of the fundamental quarks and gluon of QCD to the observed properties of hadronic interactions. A lot of work has been completed in this direction, but much remains to be done both in giving an even more solid foundation to the formalism which has been developed and in developing new frameworks in which to understand high energy reactions.

The articles in this volume aim at describing the formalism which has been developed in order to relate perturbative QCD to measurable quantities. The emphasis is placed on understanding perturbative QCD and how it relates to physical quantities rather than on detailed fits to data. It is hoped that these contributions will make the rather elaborate formalism of perturbative QCD more accessible to our theoretical colleagues in neighboring disciplines, to graduate students and to the adventurous experimenter who wants to understand exactly where QCD predictions come from and what they really mean.

At the basis of most high energy applications of QCD is factorization. Without factorization theorems, the separation of the short distance physics, perturbative QCD, from the long distance physics of observable hadrons would not be possible. The standard factorization theorems for hard processes in QCD, and their proofs, are summarized in the article of Collins, Soper and Sterman.

The article by Brodsky and Lepage deals with exclusive processes in QCD. This is a very diverse subject encompassing form factors, wide angle elastic scattering and various reactions involving nuclei. This is also a subject which has important points of contact with nonperturbative QCD and with nuclear physics.

The detailed properties of QCD jets, such as particle distributions within a jet or between several jets is given in the contribution of Dokshitzer, Khoze and Troyan. This article also describes the QCD basis for Monte Carlo models of single and multiple jet events.

One of the oldest problems in QCD, that of the behavior of small angle high energy scattering, is still not completely solved. Exactly how much of this problem can be solved purely within perturbative QCD is not completely clear at this time. 
The Pomeron problem remains one of the most challenging questions in QCD. The article by Lipatov describes the present understanding on this topic.

Infrared effects and double logarithmic terms in perturbation theory play a crucial role in many processes. For example, the transverse momentum distribution of massive $\mu$-pairs or of $\mathrm{W}$ and $\mathrm{Z}$ production in hadronic collisions can be predicted only after resuming double-logarithmic terms. Perturbative QCD is applicable to wide angle elastic scattering only because non-hard regions are suppressed, at sufficiently high energy, by the doubling logarithmic Sudakov factors. Understanding particle and multiplicity distributions in QCD jets requires good control over infrared gluon emission. These topics are discussed in the articles by Ciafaloni and by Collins.

Of course, the articles which follow are not the final word on any of these subjects. They do, however, furnish solid and fairly complete discussions as to what is known at present. We can expect factorization theorems to become more rigorous and far reaching in the future. The Pomeron and small-x problems in QCD are perhaps ripe for significant future development, solidifying our ever growing qualitative understanding of these questions. Infrared and Sudakov behavior in QCD present important technical challenges for the future. We can hope that in 10-15 years from now, significant advances and improvements will have been made in all the subjects discussed here. Nevertheless, even at that time, the present articles should remain a good introduction to the subject of perturbative QCD.

A. H. Mueller

Department of Physics Columbia University New York 


\section{CONTENTS}

Foreword

A. H. Mueller

Factorization of Hard Processes in QCD

J. C. Collins, D. E. Soper and G. Sterman

Exclusive Processes in Quantum Chromodynamics

93

S. J. Brodsky and G. P. Lepage

Coherence and Physics of QCD Jets

Yu L. Dokshitzer, V. A. Khoze and S. I. Troyan

Pomeron in Quantum Chromodynamics

L. N. Lipatov

Infrared Singularities and Coherent States in Gauge Theories

M. Ciafaloni

Sudakov Form Factors

J. C. Collins 